\title{
"A educação precisa mudar com a sociedade": uma conversa com Ana Elisa Ribeiro sobre tecnologia na escola e educação na pandemia
}

\author{
Ana Elisa Ribeiro ${ }^{\mathrm{i}}$ \\ Entrevistadores:
}

Thayane Verçosa ${ }^{\text {ii }}$

Lais Alvesii

A sólida e admirável trajetória da professora Ana Elisa Ribeiro, centrada em temas como leitura, ensino e letramento digital, produção textual e multimodalidade, justifica e explica a sua presença como entrevistada neste número da Palimpsesto. Professora Titular e pesquisadora do Departamento de Linguagem e Tecnologia do Centro Federal de Educação Tecnológica de Minas Gerais (CEFET-MG), onde atua no Programa de Pós-Graduação em Estudos de Linguagens (mestrado e doutorado), no bacharelado em Letras (Tecnologias da Edição) e no ensino médio, ela é graduada em Letras/Português, mestre em Estudos Linguísticos (Cognição, linguagem e cultura) e doutora em Linguística Aplicada (Linguagem e tecnologia) pela Universidade Federal de Minas Gerais (UFMG). Além disso, é autora dos livros Textos Multimodais (2016), Escrever, hoje (2018), Livro - Edição e tecnologias no séc. XXI (2018), e Multimodalidade, Textos e Tecnologias (2021). Para além dessa produção, a nossa entrevistada também lidera projeto de pesquisa sobre Mulheres Editoras no Brasil

\footnotetext{
i Também possui estágios pós-doutorais em Comunicação, Linguística Aplicada e Estudos Literários. Lidera o grupo de estudos (CNPq) Mulheres na Edição e é membro do GT A mulher na literatura, da ANPOLL, e da EDI-RED (estudos de edição, Madri, Espanha). É coordenadora do projeto de extensão Aula Aberta. Tem trabalhado em duas frentes: a linguística aplicada e a edição, em pesquisas que confluem sempre em questões sobre leitura e escrita. Atualmente, lidera projeto de pesquisa sobre Mulheres Editoras no Brasil (Fapemig) e o Acordo técnico-científico CEFET-MG/Academia Mineira de Letras. Sua produção pode ser encontrada no site https://anadigital.pro.br/

ii Doutoranda em Literatura Brasileira pela Universidade do Estado do Rio de Janeiro. Bolsista Capes. thayanevercosa@hotmail.com iii Mestranda em Teoria da Literatura e Literatura Comparada pela Universidade do Estado do Rio de
Janeiro (UERJ). Bolsista CAPES. lais.alvessouza@yahoo.com.br
} 
(Fapemig) e o Acordo técnico-científico CEFET-MG/Academia Mineira de Letras, o que reforça a pluralidade de sua produção.

É com muita alegria que publicamos esta entrevista com a professora Ana Elisa, que prontamente aceitou o nosso convite, sendo muito receptiva e generosa em relação às reflexões que lhe propusemos, nestes tempos tão caóticos. Aproveitamos para ressaltar que foi um prazer conhecer melhor e aprender tanto com a produção da professora, pesquisadora de assuntos tão relevantes, atuais e necessários.

\section{PALIMPSESTO}

1) Há pelo menos duas décadas, você vem coordenando projetos de pesquisa como o "Ler na tela - novos suportes para velhas tecnologias", que tematizam a leitura em suas diferentes modalidades, tendo publicado vários textos sobre o assunto. Assim, considerando sua sólida trajetória e extensa produção acadêmica, gostaríamos de saber: (a) Como se deu o seu interesse pela área? (b) Na sua opinião, quais são os autores incontornáveis, hoje, para quem deseja se aprofundar nos estudos de língua e multimodalidade?

\section{ANA ELISA RIBEIRO}

$\mathrm{Na}$ verdade, o Ler na tela foi minha dissertação de mestrado. Na virada da década de 1990 para a de 2000, o computador já estava em cena, com alguma força, em nosso país, e a internet começava a dar mostras de que também mudaria muitas de nossas práticas comunicacionais. Meu interesse nasce da minha paixão pela leitura e pela escrita. Me senti pessoalmente afetada por essas tecnologias. Sempre li e escrevi muito, e percebia, claramente, a intensidade de mudança que ocorria quando eu aprendia a escrever no Word ou a publicar na web. Seriam muitas dimensões de mudança e isso era assunto para quem estudava letramentos, na altura, embora ainda não fosse exatamente fácil encontrar parcerias de estudos e orientação nas pesquisas. Hoje, quem quer estudar multimodalidade precisa, por exemplo, ler o professor Gunther Kress, solo ou com coautores. Eu diria que são estudos de texto e de discurso sob uma abordagem multimodal. Infelizmente eles são pouco ou nada traduzidos, o que limita o acesso de muita gente às ideias desses pesquisadores e pesquisadoras, mas com paciência vamos conhecendo suas propostas e pensando as nossas. No Brasil, há muita 
gente produzindo. Sinto que cometerei alguma injustiça se citar nomes, mas é justo mencionar o prof. Francis Arthuso Paiva (UFMG), a profa. Záira Bomfante (UFES), a profa. Antônia Dilamar (UECE), a profa. Clarice Gualberto (UFMG), a profa. Vânia Barbosa (UFPI), o prof. Renato Caixeta, meu colega no CEFET-MG, entre outros tantos, em todas as partes do país, que vêm trabalhando muito nisso, inclusive com proposições.

\section{PALIMPSESTO}

2) No livro Leitura e escrita em movimento (2010), do qual você é uma das organizadoras, no capítulo publicado com Jorge Rocha, intitulado "Hipermídia e processos editoriais de produção da reportagem: o caso da 'Crônica de uma catástrofe ambiental", vocês analisam o texto "Crônica de uma catástrofe ambiental", de André Deak e Paulo Fehlauer, tratando-o como "a primeira reportagem planejada para e executada em hipermídia no Brasil" (2010, p. 230). Mais de uma década depois da publicação de tal reportagem, gostaríamos de saber como andam as reportagens hipermídias no Brasil: elas se tornaram mais recorrentes? Houve mudanças na produção de tais reportagens? Podemos pensar em alguma relação entre o isolamento social causado pela pandemia e um eventual aumento das produções hipermidiáticas?

\section{ANA ELISA RIBEIRO}

Lembro até hoje de como esse capítulo foi planejado. Escrevemos para apresentar no III Encontro Nacional sobre Hipertexto, um dos eventos itinerantes mais bacanas da época, sediado no CEFET-MG, em BH, no ano de 2009. De fato, o jornalismo é um terreno fértil para a experimentação digital, é um campo que não tem medo de aprender e de quebrar cabeça. A educação segue bem mais lenta, tentando refletir e entender, nem sempre alcançando isso. Naquela altura, não sei se estávamos exagerando. Acho que não. De fato, reportagens daquele tipo eram raras, impressionantes. Isso certamente evoluiu. O jornalismo experimentou muito e mais, de lá para cá. Não me detive nessa área e nem nesse gênero discursivo, a reportagem, mas certamente houve muitas mudanças, nesses anos todos, e as reportagens produzidas para a web ganharam novos elementos. A pandemia provavelmente trouxe novas necessidades para todos, incluindo jornalistas e leitores. Não me detive também nesse 
tipo de investigação, mas não há como escapar das mudanças, em especial na área da comunicação.

\section{PALIMPSESTO}

3) No artigo recentemente publicado, "Educação e tecnologias digitais na pandemia: ciclos de precariedade" (2021), refletindo sobre a tentativa de transposição da sala de aula física para os ambientes online, apresentados aos professores e alunos às pressas, você traça um panorama sobre o histórico de precariedade tecnológica das escolas, especialmente as públicas. Nessa reflexão, você faz uma série de perguntas que nos levam a pensar, por exemplo, sobre o nosso futuro enquanto professores e alunos, e sobre o uso (em larga medida, compulsório) da tecnologia nesse período de pandemia e dos efeitos dessa experiência. Assim, diante de questões tão relevantes, gostaríamos de saber como você responderia a alguns de seus próprios questionamentos apresentados ao longo do artigo: (a) "Demo-nos conta de nossos preconceitos e de nossas resistências relacionadas a recursos digitais, desde muito antes da pandemia?" (RIBEIRO, 2021, p. 5); (b) "De um dia para o outro, nosso espaço físico escolar foi interditado e tivemos de pôr no ar 'avatares' imperfeitos da escola, em modo virtual. Mas isso terá sido uma boa ruptura? Ou apenas uma experiência abrupta e traumática?" (Ibid., p. 6-7); e (c) "Quanto tempo tivemos de preparo para, enfim, enfrentar uma virada brusca, como a de agora, em direção às digitalidades? Alguns/mas dirão que tiveram um fim de semana; outros assumirão: tivemos três décadas. É uma contingência. E depois, o que será?" (Ibid., p. 12).

\section{ANA ELISA RIBEIRO}

Esse artigo que escrevi é derivado de uma conferência proferida no ABRALIN AO VIVO, evento on-line que a Associação Brasileira de Linguística ofereceu durante a crise sanitária. Foi uma honra estar lá. E eu fiz quase um desabafo. Era o que eu podia pensar até ali. Hoje, talvez eu possa dizer que: (a) creio que muitos e muitas de nós nos demos conta, sim, desses preconceitos; alguns os abandonamos; outros os reforçamos. Muitas resistências devem ter sido vencidas, mas a escola continua conservadora, em especial no aspecto tecnológico, para não mencionar outros. A pandemia provavelmente não terá sido um salto rumo a algo mais ajustado ao nosso tempo. (b) Certamente tivemos uma experiência abrupta e traumática. O negócio é como vamos elaborar esse trauma. Às vezes tenho a sensação de que foi uma dobra no tempo, um momento de lapso, e voltaremos a 2019 na escola. Isso me frustra profundamente. Não acho que 
tenha havido ruptura, passado mais de um ano desde minha conferência, que foi a apenas alguns meses da suspensão das aulas, naquela altura. Parece que foi apenas uma suspensão... para pôr toda a poeira embaixo do tapete de novo. (c) Nem diria mais que foi uma virada. Talvez tenha sido um "aguenta a mão", mas nada que nos leve a outro patamar, e não apenas em termos tecnológicos, mas também pedagógicos. Sonhei um pouco com isso, embora eu saiba que a tecnologia, por si, não faz milagre algum. Mas a chance de parar tudo, pensar e reconectar... poderia ter feito. Apenas para falar da minha área, a de Letras, o domínio de tecnologias digitais está nas diretrizes curriculares desde 2001, são vinte anos! Nem décadas e nem a pandemia parecem suficientes para que cheguemos ao presente. Vejam que estou bem mais pessimista hoje, depois de viver a pandemia, o ensino remoto e um ensaio às aulas presenciais.

\section{PALIMPSESTO}

4) No seu texto recentemente publicado, "Híbrido do bem, híbrido do mal", você reflete sobre as mudanças no ensino forçosamente vivenciadas por causa da pandemia do Coronavírus. Nessas reflexões, você analisa aquilo que vem sendo chamado de ensino híbrido, apresentando-o da seguinte forma: "Uma câmera fica ligada na sala, transmitindo a 'aula' para estudantes que estão em casa, enquanto o(a) professor(a) leciona para alguns(mas) estudantes que estão fisicamente na escola. $\mathrm{O}$ (a) professor(a) expõe sua matéria, cuida de ensinar aos que estão 'presentes', enquanto a câmera se mantém ligada, tal como se fosse uma simples 'câmera de segurança', certamente dividindo uma 'interação' que não atende a ninguém de forma completa. A isso talvez seja inapropriado chamar de 'híbrido'. Trata-se, a meu ver, de uma 'aula monstrenga", (RIBEIRO, 2021, p. 13). Tal cenário se tornou uma realidade nas escolas, principalmente nas particulares, uma prática comum que atravessa quase todas as séries e matérias. Diante dessa presença tão constante, perguntamos: (a) Você passou pela experiência de lecionar em tal formato? Como foi?; (b) De uma forma geral, como têm sido os relatos dos professores que estão experimentando tal formato? Há aspectos positivos?; (c) Alguns cursos de idiomas têm apresentado o modelo "híbrido" como uma novidade, uma nova realidade para os cursos de línguas, na qual o aluno é livre para escolher como vai assistir a cada aula. Considerando as interações necessárias em uma aula de língua estrangeira, qual é a sua opinião sobre isso?

\section{ANA ELISA RIBEIRO}


Graças a Deus e à autonomia da instituição em que atuo, não passei pessoalmente por esta experiência monstrenga. Conheço inúmeros(as) colegas que passaram. Todos concordamos que seja uma visão distorcida de híbrido e uma "solução" desajeitada e exploradora para oferecer aulas a estudantes em diferentes situações. A diferença é que eu posso expressar o que penso e posso pensar abertamente; nem todos os meus colegas e minhas colegas podem dizer o que pensam. Sequer podem ajudar a escola a produzir algo mais inteligente e interessante. Há muitas pessoas constrangidas e pressionadas pelo dinheiro. Minha experiência é a do ensino remoto emergencial, que funcionou melhor em alguns níveis de ensino e pior em outros, mas que vejo como a única alternativa à situação de crise. Se não fosse isso, o caso seria de não oferecer nada, como ocorreu a escolas mais precárias do que a minha. Minha próxima experiência será na sala de aula presencial, infelizmente sem qualquer melhora. Não ouvi ninguém dizer que dar aulas com uma câmera ligada, com um olho no gato e outro no peixe, era bom, positivo ou eficaz. Ouvi relatos horríveis, humilhantes. Ouvi um ou outro dizer que não ligava, que abstraía a presença daquela câmera. Acho que as pessoas aprendem de maneiras diferentes. Os pesquisadores e pesquisadoras do ensino de línguas costumam estudar questões como motivação e estilos de aprendizagem. Na língua materna isso é bem menos comum. $\mathrm{O}$ fato é que deve haver quem tenha muitas dificuldades com o ambiente das aulas presenciais, e deve haver quem tenha dificuldades no on-line. É preciso experimentar para saber. Sem isso, é tudo hipótese e preconceito. A pandemia propiciou uma experiência para muita gente. Ouvi da diretora da escola do meu filho, uma instituição privada: "teve adolescente que deslanchou, teve adolescente que paralisou". Ela se referia ao ensino remoto emergencial. Agora convenhamos: sempre houve quem deslanchasse e quem paralisasse no presencial! Mas passamos feito tratores. Há pessoas que aprendem mais lendo, estudando sozinhas, pedindo apoio apenas nas dúvidas; há pessoas que dependem mais de exposição, de explicação oral. Enfim, creio que as escolas de idiomas possam beneficiar estudantes ao dar opção. O problema é quando a escola não é honesta e está beneficiando apenas a si mesma, explorando o trabalho de professores sem vínculo, precarizando etc. Aí a questão é outra. É preciso tomar cuidado com o que é só discurso bacana para vender matrícula e o que é mesmo interesse educacional. 


\section{PALIMPSESTO}

5) No artigo "Letramento digital e ensino remoto: reflexões sobre práticas" (2020), você reflete sobre a questão do letramento digital, analisando as mudanças didáticas vivenciadas na educação em decorrência do isolamento social. Pensando sobre as escolhas das atividades para as aulas e seus impactos no novo formato, você termina sua reflexão da seguinte forma: "O importante, penso, é jamais desfigurar o que só trará impertinências, dificuldades, sofrimentos e a impressão falsa de que o problema é a tecnologia, e não as nossas más escolhas" (RIBEIRO, 2020, p. 458). Assim, considerando que a tecnologia pode nos auxiliar, como fazer boas escolhas para as aulas? Nos cursos de Licenciatura nem sempre os(as) alunos(as) são estimulados(as) a usar a tecnologia em sala de aula, algo que muitas vezes os professores também não fazem. Como podemos naturalizar nos diferentes cursos a percepção da tecnologia como uma ferramenta poderosa?

\section{ANA ELISA RIBEIRO}

Primeiro nós temos de poder fazer escolhas. Não é qualquer escola que nos dá essa condição. Liberdade, respeito, autonomia... são artigos de luxo na profissão. A nossa sociedade não confia no professor, de maneira geral. Para fazer boas escolhas é preciso pensar, estudar, conversar, trocar ideias, debater. Transferir uma aula ruim para dentro de uma tela de último tipo provavelmente não melhora em nada o ensino e a aprendizagem. Uma aula boa não depende de quantos $\mathrm{K}$ ou de quantos lúmens um projetor tem. Podemos agregar tecnologias interessantes para que aulas médias fiquem boas; podemos experimentar modos mais eficazes de ensinar algo; podemos fazer coisas com tecnologias que não fazemos analogicamente; podemos dar um upgrade em muitos aspectos da aula e da interação. Isso não quer dizer substituir tudo. $\mathrm{O}$ barato seria unir o melhor dos dois mundos, como venho dizendo e como ouvi o prof. Vilson Leffa, grande pesquisador do ensino de línguas, dizer outro dia, num evento de que participávamos on-line. Há objetivos de ensino que podemos avaliar se são melhor alcançados assim ou assado. O lance é que temos de saber o que é assim e o que é assado, entende? Pelo que ouço e estudo, os cursos de licenciatura quase nunca estimulam que se pense nas tecnologias digitais como mediação no ensino e na aprendizagem. Veja-se que a pandemia não foi fácil para ninguém. Sequer para professores e professoras recémformados! Não é curioso? Acho que isso é como uma cascata ou um efeito dominó: se 
não se pode oferecer aulas em modalidades diversas, não teremos jamais a autorização para experimentar, a liberdade de escolher e a condição de testar e avaliar, pensar sobre. Se isso não for permitido às escolas, não haverá pressão sobre a profissão, sobre os cursos de formação, sobre os estudantes. Os pais são, talvez, o elo mais conservador das escolas. As escolas precisam pressionar de volta. É importante que pais percebam que a educação precisa mudar com a sociedade. Se não existe escola que dê chance ao digital, de alguma forma e sem cinismo, para quê formar para algo que não existe? A formação precisa responder às demandas da profissão, que por sua vez traz quase nada de demandas de mudança pedagógico-tecnológica. Não sei de onde a mudança precisa vir primeiro, mas se uma coisa puxar, a outra talvez vá. Os documentos oficiais dão condição para a formação com tecnologias, mas eles são letra morta, nesse sentido. Por exemplo: não estou autorizada a oferecer disciplinas on-line no curso de graduação em Letras em que atuo. Não é culpa da instituição (só), mas dos documentos que não permitem isso. Esses documentos mudaram e o curso está levando anos e anos para se ajustar a eles. Daqui a pouco, poderemos ofertar disciplinas parcialmente on-line. Daí veremos quantos professores terão coragem ou condição de fazer isso. É uma experiência importante para os alunos, tanto como estudantes quanto como futuros docentes, quando é o caso. Não sei se estou sendo clara, mas acho que é evidente que documentos, só, não resolvem, mas pavimentam um caminho que abre possibilidades; estas, por sua vez, precisam ser efetivadas pelas pessoas, por professores de futuros professores. E assim por diante. Mas se a pessoa chegar numa escola de educação básica e nada lá sinalizar para estas possibilidades... estaremos na mesma, sempre. Alguém precisa começar.

\section{PALIMPSESTO}

6) No artigo "Duas tecnologias de um livro: alfabetizar em linguagens" (2020), publicado com a professora Amanda Ribeiro Barbosa, vocês refletem sobre o letramento e a alfabetização das crianças, a partir da análise do livro Amal e a viagem mais importante de sua vida. Comentando essa produção, vocês dizem: "Podemos perceber que Amal e a viagem mais importante de sua vida é um livro ou um projeto, a um tempo literário e informativo, e que editá-lo para os meios digital e impresso tem algumas intenções, para além da leitura literária ou de entretenimento: alcançar 
leitores/as e apoiadores/as, isto é, tratar de refugiados, no contexto atual, reúne objetivos ligados ao letramento em sentido muito mais amplo do que a fruição literária" (RIBEIRO; BARBOSA, 2020, p. 52). Considerando a proposta do livro, bem como as diferenças entre os dois formatos de leitura - como mostrado no artigo -, podemos pensar que publicações semelhantes estão se tornando mais comuns? No final do artigo, vocês comentam: "Ainda que o texto-palavra seja o mesmo, com as mesmas sequências de palavras, na mesma língua, a composição multimodal planejada e executada para cada versão transforma uma obra em pelo menos duas, do ponto de vista do produto editorial (e são vendidos e escoados de maneiras muito diferentes) e do ponto de vista da experiência de leitura" (Ibid., p. 54). Pensando em termos de práticas didáticas, como podemos levar experiências editoriais como Amal e a viagem mais importante de sua vida para as salas de aula? Poderíamos contrastar as duas versões, ressaltando as diferenças entre ambas? Seria melhor escolher apenas uma delas?

\section{ANA ELISA RIBEIRO}

Primeiro preciso externar que escrever com Amanda é um bálsamo. Ela é uma professora sensível e antenada, séria e apaixonada pela docência. É pedagoga e poeta. À pergunta eu diria que "escolher" é uma palavra-chave. Ter versões é riqueza. E isso é altamente multimodal. E o que é multimodal não é substituível. Digo: um dos pontos centrais da abordagem multimodal é que cada modo semiótico só consegue expressar parcialmente os sentidos. Por isso, ter duas versões significa ter duas obras, no sentido de que vamos ler diferente, usando recursos diferentes, conciliando e inconciliando em combinações diversas, facilitando aqui e dificultando ali, cada coisa a seu modo. Não há por que excluir, se é possível ofertar em profusão. Levar essas experiências ao leitor e à leitora é tornar a leitura mais rica, seja em aula ou não. Ressaltar as diferenças pode ser interessante, assim como ressaltar as semelhanças. Importante mesmo é dar a ver que cada um é um, a seu modo, do seu jeito, e cada um se lê assim ou assado. Se não formos pobremente obrigados a escolher uma das versões, eu diria que sempre deveríamos ter todas.

\section{PALIMPSESTO}

7) No ano passado, no número 34 da Palimpsesto, tivemos a oportunidade entrevistar a professora Carla Coscarelli. ${ }^{1}$ Para formular as perguntas, lemos, dentre outros materiais, dois artigos escritos em parceria com você: "O que dizem as matrizes de habilidades 
sobre a leitura em ambientes digitais" (2010) e "Leitura e ensino: por avaliações que levem (mesmo) os ambientes digitais em consideração" (2020). Sobre este, a partir de uma passagem, fizemos uma pergunta sobre fake news. Considerando a relevância e a atualidade do tema, e a oportunidade de entrevistá-la, repetiremos a questão. Em "Leitura e ensino: por avaliações que levem (mesmo) os ambientes digitais em consideração" (2019), ao refletir sobre o crescimento e a relevância da leitura em ambientes digitais, bem como justificar a necessidade de que as práticas de leitura em tais ambientes sejam incluídas nos currículos escolares, vocês refletem: "A maioria dos adolescentes parece nunca ter aprendido o que fazer para julgar a confiabilidade das informações que encontra na internet [...], uma vez que não usam estratégias eficientes para avaliar esse quesito" (COSCARELLI; RIBEIRO, 2020, p. 103). Em que medida esta lacuna educacional se relaciona com a difusão das fake news e do seu impacto negativo no cotidiano das pessoas? Além da inclusão dessas práticas nos currículos escolares, podemos pensar em outras medidas que auxiliem na construção da capacidade de "julgar a confiabilidade das informações"?

\section{ANA ELISA RIBEIRO}

Vejam que coisa séria isso é. E não diz respeito apenas a crianças e adolescentes, embora estivéssemos, eu e Carla, ocupadas das salas de aula. Adultos são enganados por notícias falsas todos os dias. Ler é algo para a vida toda de uma pessoa. E socialmente a escola é cobrada por isso, tem essa atribuição. A Carla (outra dessas pessoas que são bênçãos em nossas vidas) é muito mais especialista nisso do que eu, mas posso dizer que "ensinar a ler" é uma expressão que muda, que tem sua carga alterada, à medida que a sociedade também muda e se complexifica. Uma de nossas tarefas hoje é ensinar crianças e jovens a lidarem com as tecnologias e as redes, os textos que circulam por todo lado. Para isso, nós temos de ficar craques nisso. Notícias falsas têm impacto negativo, claro. Conheço gente supostamente esclarecida, estudada, com formação superior, que diz coisas impressionantes sobre os benefícios de não se vacinar contra covid ou outras doenças. No caso, isso deixa de ser apenas uma incompetência para ler e passa a outro patamar de problema. Enquanto podemos ensinar a ler, a pesquisar, a fazer julgamentos que levem a uma melhor compreensão dos textos, da vida... será bom.

\section{PALIMPSESTO}


8) Um de seus projetos atuais de pesquisa, iniciado em 2017, intitulado "Mulheres que editam: um mapeamento preliminar no Brasil", é apresentado da seguinte forma: "O projeto tem o objetivo de mapear mulheres editoras de livros literários e de outros segmentos, no passado e no presente, no Brasil" (Fonte: Currículo Lattes). Para além dele, há outro projeto de pesquisa seu, iniciado em 2018, intitulado "Mulheres editoras de livros literários em Minas Gerais". Assim, gostaríamos de saber: há uma relação entre os dois projetos? Qual? De alguma forma eles se relacionam ao "Acordo de cooperação técnico-científica POSLING CEFET-MG e Academia Mineira de Letras" (projeto de pesquisa iniciado em 2020)? Os mencionados projetos já produziram materiais?

\section{ANA ELISA RIBEIRO}

Depois de estudar escritoras brasileiras, em especial algumas muito famosas e outras que consideramos "apagadas" ou esquecidas, me toquei de que nada se dizia sobre as mulheres editoras, isto é, figuras, profissionais ou não, que selecionam, editam, publicam livros, seus e de outras. É uma lacuna interessante, um espaço de pesquisa incrível. Os dois projetos são muito semelhantes, diferenciando-se pela abrangência geográfica: um é em Minas, o outro é nacional. E é claro que vou executando tudo meio misturado, porque esse tipo de pesquisa é difícil, as fontes são escassas, silenciadas e silenciosas também, então vou pesquisando à medida que encontro, que farejo, que escavo. O Acordo de Cooperação com a Academia Mineira de Letras é algo de que me orgulho. Primeiro porque a AML é um acervo riquíssimo e nós temos preferência, dentro do Acordo, para pesquisar lá; segundo porque a burocracia e a morosidade institucionais do CEFET-MG para selar este Acordo foram tão imensas... que nossa persistência ficou mais que provada (e comprovada), é mesmo algo que inibe as iniciativas; terceiro que já temos lá, a partir do Acordo, pesquisadores de vários níveis (doutores, doutorandos, mestrandos, iniciação científica e voluntários) que não apenas ajudam a tratar o acervo de mais de trinta e cinco mil itens que a AML guarda, mas também que leem, estudam e revelam elementos importantes de nossa história cultural, literária e editorial. Os projetos todos já geraram vários artigos publicados em revistas científicas nacionais e estrangeiras, capítulos em diversos livros, eventos, conferências dentro e fora do Brasil, conexões com grupos de pesquisa aqui e no exterior, com o GT A mulher na literatura, da ANPOLL, assim como o fortalecimento de nosso grupo de pesquisa, o Mulheres na Edição, que conta com mais de duzentas pessoas cadastradas e participantes. Publiquei, em 2020, o livro de acesso livre e gratuito Subnarradas - 
mulheres que editam, ${ }^{2}$ pela adorável Zazie Edições, mas nossa produção mais recente é o livro Prezada editora, mulheres no mercado editorial brasileiro, ${ }^{3}$ lançado em 2021 pela coleção Pensar Edição, das editoras Moinhos e Contafios. Feliz e infelizmente, sempre haverá o que dizer sobre o tema.

\section{Referências}

COSCARELLI, Carla Viana; RIBEIRO, Ana Elisa. Leitura e ensino: por avaliações que levem (mesmo) os ambientes digitais em consideração. Texto Digital, Florianópolis, v. 15, n. 2, p. 101-129, 2020. Disponível em: <https://periodicos.ufsc.br/index.php/textodigital/article/view/18079288.2019v15n2p101/42347>. Acesso em: 16 out. 2020.

RIBEIRO, Ana Elisa. Educação e tecnologias digitais na pandemia: ciclos da precariedade. Cadernos de Linguística, v. 2, n. 1, p. 01-16, 2021.

.Híbrido do bem, híbrido do mal. Revista Bis, n. 55, p. 12-13, jul.-set. 2021.

.; ROCHA, Jorge. Hipermídia e processos editoriais de produção de reportagem: o caso da "Crônica de uma catástrofe ambiental”. In: ___.; Ana Maria Nápoles Villela; SOBRINHO, Jerônimo Coura; SILVA, Rogério Barbosa da. Leitura e escrita em movimento. São Paulo: Editora Peirópolis, 2010. p. 229-238.

Letramento digital e ensino remoto: reflexões sobre práticas. Debates em Educação, v. 12, n. 2, p. 446-460, 2020.

.; BARBOSA, Amanda Ribeiro. Duas tecnologias de um livro: alfabetizar em linguagens. Revista Brasileira de Alfabetização, n. 13, p. 46-56, 2020.

\footnotetext{
1 Disponível em: https://www.e-publicacoes.uerj.br/index.php/palimpsesto/article/view/56238/36195. Acesso em: 27 out. 2021.

${ }^{2}$ Livro disponível em: https://zazie.com.br/wp-content/uploads/2021/05/ANA-ELISA-RIBEIRO-7.pdf. Acesso em: 4 dez. 2021.

3 Livro disponível em: https://editoramoinhos.com.br/loja/prezada-editora-mulheres-no-mercadoeditorial-brasileiro/. Acesso em: 4 dez. 2021.
} 


\title{
"Education needs to change along with society": a conversation with Ana Elisa Ribeiro about technology in school and teaching in pandemic times
}

\author{
Ana Elisa Ribeiro ${ }^{\mathrm{i}}$ \\ Entrevistadores:
}

Thayane Verçosa ${ }^{\text {ii }}$

Lais Alvesii

Professor Ana Elisa Ribeiro's solid and admirable trajectory focused on topics such as reading, teaching and digital literacy, text production and multimodality, justifies and explains her presence as an interviewee in this issue of Palimpsesto. Ribeiro is a professor and researcher at the Language and Technology Department at the Centro Federal de Educação Tecnológica de Minas Gerais (CEFET-MG), where she is active in the Postgraduate Program in Language Studies (master's and doctorate), in the undergraduate program in Languages (Publishing Technologies), and secondary education. She holds a degree in Portuguese Language from the Federal University of Minas Gerais (UFMG), a master's degree in Linguistic Studies (Cognition, Language, and Culture), and a doctorate in Applied Linguistics (Language and Technology) from the same university. In addition, she is the author of the books Textos Multimodais (2016), Escrever, hoje (2018), Livro - Edição e tecnologias no séc. XXI (2018), and Multimodalidade, Textos e Tecnologias (2021). Besides this production, our interviewee

\footnotetext{
${ }^{\mathrm{i}}$ Professor Ana Elisa Ribeiro has post-doctoral internships in Communication, Applied Linguistics, and Literary Studies. She leads the study group Women in Publishing (CNPq) and is a member of the WG Women in Literature of ANPOLL and EDI-RED (Publishing Studies, Madrid, Spain). In adittion, she is a coordinator of the Aula Aberta extension project. Ribeiro has worked on two fronts: Applied Linguistics and Editing, with researches that always converge on questions about reading and writing. She is currently leading a research project on Women Publishers in Brazil (Fapemig) and the Acordo TécnicoCientífico CEFET-MG/Academia Mineira de Letras. Her productions can be found at http://anadigital.pro.br/.

ii PhD Student in Literary Studies at Rio de Janeiro State University (UERJ, in Portuguese). CAPES Scholar. thayanevercosa@ hotmail.com

iii Master's Student in Literary Studies at Rio de Janeiro State University (UERJ, in Portuguese). CAPES Scholar. lais.alvessouza@yahoo.com.br
} 
also leads a research project on Women Publishers in Brazil (Fapemig) and the Acordo Técnico-Científico CEFET-MG/Academia Mineira de Letras, which reinforces the plurality of her production.

We are glad to publish this interview with Professor Ana Elisa, who promptly accepted our invitation, being very receptive and generous concerning the questions we proposed to her in these chaotic times. We take this opportunity to emphasize that it was a pleasure to get to know her better and learn so much from her production, a researcher of such relevant, current, and necessary subjects.

\section{PALIMPSESTO}

1) For at least two decades, you have been coordinating research projects related to reading in its different modalities, such as "Ler na tela - novos suportes para velhas tecnologias" ["Reading on the screen - new devices for old technologies"], and publishing several texts on the subject. Thus, considering your solid background and extensive academic production, we would like to know: (a) How did your interest in the area come about? (b) In your opinion, which are the unavoidable authors today for those who wish to deepen their knowledge of language and multimodality studies?

\section{ANA ELISA RIBEIRO}

Actually, "Ler na tela" was my master's dissertation. At the turn of the 1990s to the 2000s, the computer was already on the scene with some force in our country, and the internet was beginning to sign that it would also change many of our communication practices. My interest was born out of my passion for reading and writing. I felt personally affected by these technologies. I always read and wrote a lot, and I could clearly see the intense transformation when I learned to write in Microsoft Word or publish on the web. There would be many dimensions of change, which was a matter for those who studied literacy, although it was not exactly easy to find study partnerships and research supervision back then. Today, those who want to study multimodality need, for example, to read Professor Gunther Kress, solo or with coauthors. I would say that those are studies on text and discourse under a multimodal approach. Unfortunately, there are few or no translations into Portuguese at all of these studies, limiting the access for many people. However, with patience, we get to know 
their proposals and think about ours. In Brazil, there are many people producing studies in this area. I feel I may be somewhat unjust if I cite only some names. Nevertheless, it is important to mention Professors Francis Arthuso Paiva (UFMG), Záira Bomfante (UFES), Antônia Dilamar (UECE), Clarice Gualberto (UFMG), Vânia Barbosa (UFPI), Renato Caixeta - my colleague at CEFET-MG -, among many others, in all parts of the country, who have been working hard on this, including proposing their own ideas and theories.

\section{PALIMPSESTO}

2) In the book Leitura e escrita em movimento [Reading and writing in motion] (2010), of which you are one of the organizers, in the chapter entitled "Hipermídia e processos editoriais de produção da reportagem: o caso da 'Crônica de uma catástrofe ambiental"' ["Hypermedia and editorial processes of report production: the case of 'Chronicle of an environmental catastrophe"'], you and Jorge Rocha analyze the text "Crônica de uma catástrofe ambiental" ["Cronicle of an environmental catastrophe"], by André Deak and Paulo Fehlauer. You treat it as "the first report for and executed in hypermedia in Brazil" (2010, p. 230). More than a decade after the publication of Deak and Fehlauer's report, we would like to know how hypermedia reporting is going in Brazil: has it become more recurrent? Have there been any changes in the production of such reports? Can we think of any relation between the social isolation caused by the pandemic and an eventual increase in hypermedia productions?

\section{ANA ELISA RIBEIRO}

I remember to this day how this chapter was planned. We wrote it to a presentation at the III Encontro Nacional sobre Hipertexto [III National Meeting on Hypertext], one of the most exciting events, held at CEFET-MG, in Belo Horizonte, in 2009. In fact, journalism is a fertile ground for digital experimentation. It is a field that does not fear to learn and to rack its brain. The field of education proceeds in a much slower way, trying to reflect and understand, not always achieving a complete understanding. I do not know if we were exaggerating at that time - I do not think so. In fact, reports of that type were rare, impressive. But this situation has certainly evolved. Journalism has experimented a lot and more since then. I did not retain myself in this area or this discursive genre - the report -, but there were many changes in all these 
years, and the reports produced for the web gained new elements. The pandemic has probably brought new necessities for everyone, including journalists and readers. I did not dwell on this type of research either, but there is no escaping the changes, especially in Communication.

\section{PALIMPSESTO}

3) In "Educação e tecnologias digitais na pandemia: ciclos de precariedade" ["Education and digital technologies in the pandemic: cycles of precariousness"] (2021), you reflect on the attempt to transpose the physical classroom to online environments, which were presented to teachers and students in a hurry. You trace an overview of the history of the technological precariousness of schools, especially public ones. In your reflection, you ask a series of questions that makes us think, for example, about our future as teachers and students and the (to a great extent, compulsory) use of technology during the pandemic and the effects of this experience. Thus, we would like to know how you would answer some of your own questions presented throughout the article: (a) "Have we realized our prejudices and resistances related to digital resources since long before the pandemic?" (RIBEIRO, 2021, p. 5); (b) "From one day to the other, our physical school space was interdicted, and we had to put on online 'avatars' of the school. However, was that a good rupture? Or just an abrupt and traumatic experience?" (p. 6-7); and (c) "How long have we had to prepare ourselves to finally face an abrupt turn, like now, towards digitality? Some will say they had a weekend; others will assure us that we had three decades. It is a contingency. And then, what will it be?" (p. 12).

\section{ANA ELISA RIBEIRO}

This article I wrote derived from a conference given at the ABRALIN AO VIVO [ABRALIN LIVE], an online event the Association offered during the pandemic. It was an honor to be there. This was almost an outburst on my part. That was all I could think of then. Today, maybe I can say that: (a) I believe that many of us have indeed become aware of these prejudices; some of us have abandoned them; others have reinforced them. Many resistances may have been overcome, but the school continues to be conservative, especially in the technological aspect, not to mention others. The pandemic will probably not have been a step towards something more in tune with our times. (b) We certainly had an abrupt and traumatic experience. The point is how we will elaborate on that trauma. Sometimes I feel it was a time warp, a moment of lapse, 
and we will be back to school in 2019. This state of affairs frustrates me deeply. More than a year since my conference - which was only a few months away from the suspension of classes [in Brazil schools] -, I do not believe there has been a breakthrough. It seems like it was just a suspension... to put all the dust under the rug again. (c) I would not even say it was a turnaround anymore. Maybe it was a "hold our hands", but nothing that will take us to another level, not only in terms of technology but also pedagogically. I fancied about it a little, although I know that technology, by itself, does no miracle. However, the chance to stop everything, think, and reconnect could have done miracles. Speaking only about my area, that of Languages, the mastery of digital technologies has been in the curriculum guidelines since 2001 - twenty years. Not decades, not even the pandemic seem enough for us to get to the present. See that I am much more pessimistic today after living through the pandemic, remote teaching, and a rehearsal for face-to-face classes.

\section{PALIMPSESTO}

4) In your recently published text "Híbrido do bem, híbrido do mal" ["Good hybrid, evil hybrid"] (2021), you reflect on the changes that have been forced upon teaching due to the Coronavirus pandemic. In your reflection, you analyze what has been called "hybrid teaching," presenting it as follows: "A camera stays on in the classroom, broadcasting the 'class' to students at home, while the teacher is lecturing some students who are physically at school. The teacher presents the subject, takes care of teaching those who are 'present.' The camera keeps on, as if it were a simple 'security camera,' certainly sharing an 'interaction' that serves no one completely. It is perhaps inappropriate to call this a 'hybrid' teaching. In my view, it is a 'monster class"' (RIBEIRO, 2021, p. 13). Such a scenario has become a reality in schools, especially private ones, a common practice that cuts across almost all grades and subjects. Faced with such a constant presence, we ask: (a) Have you experienced teaching in such a format? How was it?; (b) In general, what have been the reports from teachers who are experimenting with this format? Are there any positive aspects? (c) Some language schools have presented the "hybrid" model as a novelty, a new reality for free language courses, in which the student is free to choose how s/he will attend each class. Considering the interactions required in a foreign language class, what is your opinion about this?

\section{ANA ELISA RIBEIRO}


Thanks to God and the autonomy of the institution where I work, I have not personally gone through this monstrous experience. I know countless colleagues who have, although. We all agree that it is a distorted view of hybrid teaching, it is an exploitative "solution" to offer classes to students in different situations. The difference is that I can express what I think and think openly; not all my colleagues can say what they think. They cannot even help the school produce something more intelligent and interesting. Too many people have been embarrassed and pressured for money. My experience is that of emergency remote teaching, which has worked better at some levels of education and worse at others, but I see it as the only alternative to the situation brought about by the crisis. Otherwise, the case would be to offer nothing at all, as has occurred to schools more precarious than mine. My next experience will be in the face-to-face classroom, unfortunately without any improvement. I have not heard anyone say that teaching with a camera on, with one eye on the cat and the other on the fish, was good, positive, or effective. On the contrary, I heard horrible, humiliating reports. I heard one or another colleague saying that they did not care, that they abstracted from the presence of the camera. I believe people learn in different ways. Foreign-language teaching researchers often study issues such as motivation and learning styles. This situation is much less common concerning the mother tongue [Portuguese]. The fact is that there must be some who have much difficulty with the face-to-face classroom environment, and there must be some who have difficulty online. You have to experience both modes to know. Without these experimentations, everything is hypothesis and prejudice. The pandemic provided an experience for many people. I heard from the principal of my son's school, which is a private institution: "there were teenagers who took off, and there were teenagers who got stuck". She was referring to emergency remote education. Now let us face it: there have always been those who have succeeded and those who have stopped in on-site teaching! But we go through this situation like tractors. Some people learn more by reading, studying alone, asking for support only when in doubt; others depend more on exposure and oral explanation. In short, I believe that language schools can help students by giving them options. The problem is when the school is not honest and is only benefiting itself, exploiting the work of teachers without a contract, making it more precarious, etc. - this 
is a different matter. It is necessary to identify what is mere sales talk from what is genuinely educational interest.

\section{PALIMPSESTO}

5) In the article "Letramento digital e ensino remoto: reflexões sobre práticas" ["Digital literacy and remote teaching: reflections on practices"] (2020), you consider the issue of digital literacy, analyzing the didactic changes experienced in education as a result of social isolation. You end your reflection thinking about the choices of activities for the classes and their impacts in the new format: "The important thing, I believe, is never to disfigure what will only bring impertinence, difficulties, suffering, and the false impression that the problem is the technology, and not our bad choices" (RIBEIRO, 2020 , p. 458). Hence, considering that technology can help us, how to make good choices for classes? Students are not always stimulated to use technology in undergraduate courses, which teachers often do not do either. How can we naturalize the perception of technology as a powerful tool in different courses?

\section{ANA ELISA RIBEIRO}

First, we must be able to make choices - not any school gives us this condition. Freedom, respect, and autonomy are luxury terms in our profession. In general, society does not trust teachers. It is necessary to think, study, talk, exchange ideas, and debate to make good choices. Transferring a bad class into a screen probably does not improve teaching and learning at all. A good class does not depend upon how many K's or how many lumens a projector has. We can add interesting technologies to make good classes out of average classes; we can experiment with more effective ways of teaching something; we can do things with technologies that we cannot do analogically; we can upgrade many aspects of class and interaction - all this does not mean replacing everything. The best thing would be to unite the best of both worlds, as I have been saying and as I heard Professor Vilson Leffa, a great language teaching researcher, say in an event we were attending online the other day. There are teaching objectives that we can evaluate if they are better achieved this way or that way. The thing is that we must know what is this way and what is that way. I hear and study that undergraduate courses seldom encourage students to think about digital technologies as mediation in the teaching and learning processes. We see that the pandemic was not easy for anyone 
- not even for newly graduated teachers! Is not this curious? I think this situation is like a cascade or a domino effect: if you cannot offer classes in different modalities, we will never have the authorization to experiment, the freedom to choose, and the conditions to test and evaluate, to think about it. If schools are not allowed to do this, there will be no pressure on the teachers, on undergraduate courses, on students. Parents are perhaps the most conservative link in schools. Schools need to push back. Parents need to realize that education does need to change with society. If no school somehow gives the digital tools a chance, without cynicism, why do we graduate future teachers for something that does not exist? The training needs to respond to the demands of our profession, which in turn brings almost no demands for pedagogical-technological changes. I do not know where the changes need to be applied first, but if one thing pulls, the other might come. The official documents provide conditions for training with technologies, but they are dead letters in this sense. For example, I am not authorized to offer online courses in the undergraduate course where I work. It is not the institution's fault only, but the official documents that do not allow this situation are also to blame. These documents have changed, and the course is taking years and years to adjust to them. Soon, we will be able to offer partially-online courses. Then we will see how many professors will have the courage or condition to do this. It is an important experience for the students, both as students and as future teachers, if that is the case. I do not know if I am making myself clear, but I do think it is evident that documents alone do not solve the case, but they pave a path that opens possibilities; these possibilities, in turn, need to be made effective by people, by teachers of future teachers. And so on. However, if a person arrives at a primary-education school and encounters no room for these possibilities, we will always be the same. Someone has to start the process.

\section{PALIMPSESTO}

6) In the article "Duas tecnologias de um livro: alfabetizar em linguagens" ["Two technologies in a book: literacy in languages"], written in partnership with professor Amanda Ribeiro Barbosa, you reflect on literacy and children literacy, based on the analysis of the book Amal e a viagem mais importante de sua vida [Amal and the most important journey of her life, by Carolina Montenegro]. Some of your comments are: "We can perceive that Amal e a viagem mais importante de sua vida is a book or a 
project literary and informative at the same time. Its edition for digital and printed media has some intentions beyond literary reading or entertainment: to reach readers and supporters, that is, to deal with refugees in the current context brings together objectives linked to literacy in a much broader sense than literary fruition" (RIBEIRO; BARBOSA, 2020, p. 52). Considering the book's purpose and the differences between the two reading formats - as shown in the article -, can we think this type of publication is becoming more common? At the end of the article, you comment: "Even though the text-word is the same - with the same word sequences, in the same language -, the multimodal composition planned and executed for each version transforms one work into at least two from the point of view of the publishing product (and they are sold and marketed in very different ways) and from the point of view of the reading experience" (p. 54). Thinking in terms of teaching practices, how can we bring into the classroom publishing experiences like Amal e a viagem mais importante de sua vida? Could we contrast the two versions by highlighting the differences between them? Would it be better to choose only one of them?

\section{ANA ELISA RIBEIRO}

First, I must say that writing with Amanda is a balsam. She is a sensitive and attentive teacher, serious and passionate about teaching. She is a pedagogue and a poet. To your question, I would answer that "choose" is a keyword - to have versions and options is wealth. And that is highly multimodal - and what is multimodal is not replaceable. One of the central points of the multimodal approach is that each semiotic mode can only partially express the meanings. Therefore, having two versions means having two works in the sense that we will read differently, using different resources, conciliating and unconciliating them in diverse combinations, facilitating here and hindering there, each thing in its own way. There is no reason to exclude options if it is possible to offer profusion. To bring these experiences to the reader is to make a richer reading, whether in class or not. Highlighting the differences can be interesting, as well as highlighting the similarities. The important thing is to show that each one is a person, in her or his own way, and each one of us reads like this or like that. If we are not poorly obliged to choose one of the versions, I would say that we should always have all of them.

\section{PALIMPSESTO}


7) Last year, in Palimpsesto's issue 34, we had the opportunity to interview Professor Carla Coscarelli. ${ }^{1}$ To formulate our questions, we read, among other materials, two articles she wrote in partnership with you: "O que dizem as matrizes de habilidades sobre a leitura em ambientes digitais" ["What do skill matrices say about reading in digital environments"] (2010) and "Leitura e Ensino: por avaliações que levem (mesmo) os ambientes digitais em consideração" ["Reading and teaching: for assessments that (really) take digital environments into account"] (2019). About the latter, we asked her a question about fake news. We believe this topic to be timeliness and of great relevance, and as we now have the opportunity to have you as our interviewee, we will repeat the question. In your article, you consider the growth and relevance of reading in digital environments and justify the need for reading practices in such settings to be included in school curricula: "Most teenagers seem never to have learned how to judge the reliability of the information they find on the internet [...] since they do not use efficient strategies to evaluate this question" (COSCARELLI; RIBEIRO, 2020, p. 103). To what extent this educational gap is related to the diffusion of fake news and its negative impact on people's daily lives? Besides including these practices in school curricula, can we think of other means to help build the ability to "judge the reliability of information"?

\section{ANA ELISA RIBEIRO}

Look how serious this is. And it does not concern only children and teenagers, although Carla and I were referring to our classrooms in this article. Fake news deceives adults every day. Reading is something for a person's whole life. Socially, the school is charged for this, the school has this assignment. Carla (another of those people who are blessings in our lives) is much more of an expert on this than I am, but I can say that "teaching how to read" is an expression that changes, that has its meaning altered, as a society also changes and becomes more complex. One of our tasks as teachers today is to teach children and young people to deal with the technologies and networks, the texts that circulate everywhere. To do this, we have to become adept at it. Fake news has a negative impact, of course. I know supposedly enlightened, college-educated people who say absurd things about the benefits of not getting vaccinated against Covid or other diseases. In this case, it is no longer just incompetency to read, but another level of problem. As long as we can teach how to read, research, and make judgments that lead to a better understanding of texts, of life, it will be good. 


\section{PALIMPSESTO}

8) Your current research project, "Mulheres que editam: um mapeamento preliminar no Brasil" ["Women who edit: a preliminary mapping in Brazil"], started in 2017, is presented as follows: "The project aims to map women publishers of literary books and other segments in Brazil of the past and present" (source: Lattes curriculum). In addition to it, there is another project of yours, started in 2018: "Women publishers of literary books in Minas Gerais." Thus, we would like to know: is there a relation between the two projects? What is it? Are they somehow related to the "Acordo de cooperação técnico-científica POSLING CEFET-MG e Academia Mineira de Letras" ["POSLING CEFET-MG and Academia Mineira de Letras' technical-scientific cooperation agreement"] (a research project started in 2020)? Have these projects already produced any materials?

\section{ANA ELISA RIBEIRO}

After studying Brazilian women writers, especially very famous ones and others considered "erased" or forgotten, I realized that nothing was said about women editors, that is, figures - professional or not - who select, edit, and publish books - their own and others'. It is an interesting gap, an incredible research space. Both projects are very similar, differentiated only by their geographical scope: one is about Minas [Gerais, a Brazilian southeast state], the other has a national range. Of course, I am doing both projects a bit mixed up, because this type of research is complex - sources are scarce, silenced and silent. So, I do my research as I find, as I sniff, as I dig. The Acordo de Cooperação com a Academia Mineira de Letras is something that I am proud of. First because AML [the Agreement's acronym in Portuguese] is a very rich collection, and we have a preference, within the Agreement, to do research there; second, because the institutional bureaucracy and slowness of CEFET-MG to seal this Agreement were so immense that our persistence has been more than proven, it is indeed something that inhibits initiatives; third, for we already have there, as from the Agreement, research from various levels (PhDs, PhD students, Master students, undergraduate students, and volunteers) that not only help to maintain the more than thirty-five thousand items that AML keeps, but also that read, study and reveal important elements of our cultural, literary and editorial history. The projects have generated several articles published in national and foreign journals, chapters in several books, events, conferences in Brazil and abroad, connection with research groups here and abroad, with ANPOLL'S Work 
Group A mulher na literatura [Women in literature], as well as strengthening our research group, Mulheres na Edição [Women in Publishing], which has more than two hundred registered participants. In 2020, I published the free, open-access book Subnarradas - mulheres que editam ${ }^{2}$ [Undernarrated - women who edit], by the lovely Zazie Edições. However, our most recent production is the book Prezada editora: mulheres no mercado editorial brasileiro ${ }^{3}$ [Dear publisher: women in the Brazilian publishing market], published in 2021 by the collection Pensar Edição, from the publishers Moinhos and Contafios. Fortunately and unfortunately, there will always be something to say on the subject.

\section{References}

COSCARELLI, Carla Viana; RIBEIRO, Ana Elisa. Leitura e ensino: por avaliações que levem (mesmo) os ambientes digitais em consideração. Texto Digital, Florianópolis, v. 15, n. 2, p. 101-129, 2020. Disponível em: <https://periodicos.ufsc.br/index.php/textodigital/article/view/18079288.2019v15n2p101/42347>. Acesso em: 16 out. 2020.

RIBEIRO, Ana Elisa. Educação e tecnologias digitais na pandemia: ciclos da precariedade. Cadernos de Linguística, v. 2, n. 1, p. 01-16, 2021.

Híbrido do bem, híbrido do mal. Revista Bis, n. 55, p. 12-13, jul.-set. 2021.

; ROCHA, Jorge. Hipermídia e processos editoriais de produção de reportagem: o caso da "Crônica de uma catástrofe ambiental". In: ; Ana Maria Nápoles Villela; SOBRINHO, Jerônimo Coura; SILVA, Rogério Barbosa da. Leitura e escrita em movimento. São Paulo: Editora Peirópolis, 2010. p. 229-238.

Letramento digital e ensino remoto: reflexões sobre práticas. Debates em Educação, v. 12, n. 2, p. 446-460, 2020.

; BARBOSA, Amanda Ribeiro. Duas tecnologias de um livro: alfabetizar em linguagens. Revista Brasileira de Alfabetização, n. 13, p. 46-56, 2020.

\footnotetext{
1 Available on: https://www.e-publicacoes.uerj.br/index.php/palimpsesto/article/view/56238/36195. (Accessed on October 27 $7^{\text {th }}, 2021$ )

2 Book avaliable on: https://zazie.com.br/wp-content/uploads/2021/05/ANA-ELISA-RIBEIRO-7.pdf. (Acessed on December 4th, 2021)

${ }^{3}$ Book avaliable on: https://editoramoinhos.com.br/loja/prezada-editora-mulheres-no-mercado-editorialbrasileiro/ (Acessed on December 4th, 2021)
} 